\title{
41. Immunologic Consequences and Considerations of the Laparoscopic Approach
}

\author{
Daniel L. Feingold \\ Joseph Carter \\ Richard L. Whelan
}

The introduction of advanced laparoscopic operations in the early 1990s led to a close examination of the immunologic consequences of both open and closed surgical methods. This scrutiny has significantly increased our understanding of the impact of surgery on the body and will, it is hoped, lead to new perioperative pharmacologic therapies that will lessen the deleterious immunologic effects of all types of surgery. Before the laparoscopic era, it had been well established that major open surgery is associated with the temporary suppression of a variety of cells that are involved with both innate and specific immunity, including lymphocytes, neutrophils, monocytes, and macrophages. Interactions between cells, chemotaxis, expression of surface antigens, the ability to produce cytokines, and other functions of these immune system cells are negatively influenced by open surgical trauma. Further, the ability to mount a positive response to a delayed-type hypersensitivity (DTH) recall antigen challenge is suppressed after surgery [1-5].

The relative contribution of each part of an abdominal procedure (abdominal wall access incision versus intraabdominal dissection and resection) to postsurgical immunosuppression had not been assessed before the advent of advanced laparoscopic methods. The results of recent studies suggest that the method of entry into the abdomen is the most important determinant of postoperative immune function. Minimally invasive methods, for a variety of immune parameters, have been shown to be associated with significantly better preserved function when compared to the equivalent open procedure. Of note, in many cases the differences are small and short lived, on the order of a day, and sometimes less for several variables. For a number of parameters no differences have been noted.

It should be realized that the clinical significance, if any exists, of the immune function differences that have been found has not been determined. Better preserved postoperative cell-mediated immune function, in theory, may have an impact on the rate of infections and, possibly, tumor recurrence rates and survival.

This chapter reviews the available literature in this area and also speculates on how our improved understanding of surgery's impact on the immune system may lead to novel perioperative treatments in the future. 


\section{A. Delayed-Type Hypersensitivity}

One of the simplest methods of evaluating immune function is via delayedtype hypersensitivity (DTH) testing. The ability to mount a DTH response to an intradermally injected antigen to which the subject has been previously exposed verifies that several important elements of the immune system are functioning. A positive response, as indicated by the development of an indurated area at the site of injection, requires (1) that the antigen be processed and presented to a specific CD4+ T lymphocyte by an antigen-presenting cell (APC), (2) activation and replication of the CD4+ T lymphocyte and cytokine elaboration, and (3) accumulation of "effector" cells as well as deposition of fibrin the challenge site. In the perioperative setting, serial DTH challenges, done both before and after surgery, provide the observer with some idea of the functional status of the immune system at different time points and permit comparison of postoperative responses to the patient's baseline preoperative response. Although a diminished DTH reaction is believed to correlate with a decrease in the functional status of the cell-mediated immune system, thus far it has not been demonstrated that patients with significantly decreased postoperative DTH responses have an increased rate of complications.

In mice, it has been shown that both sham laparotomy and open bowel resection via a full laparotomy are associated with a significant decrease in the size of the DTH responses for at least 5 days after surgery. The equivalent laparoscopic procedures, in contrast, were associated with significantly better preserved DTH responses [7, 8]. Two human DTH studies have been done, a cholecystectomy and a colectomy study; both were fairly small and nonrandomized. Both studies demonstrated that the open procedure was associated with a significant drop in the size of the response whereas the minimally invasive groups had smaller nonsignificant decreases [9, 10]. These studies, and others, suggest that early after surgery cell-mediated immune function is better preserved after laparoscopic procedures.

\section{B. Lymphocyte Proliferation}

Inherent to cellular immunity is lymphocyte function, which can be assessed in a number of ways. One method is to perform lymphocyte proliferation assays (LPA). In an LPA, lymphocytes from peripheral blood samples are exposed, in vitro, to a mitogen such as lipopolysaccharide, and their degree of proliferation measured. A decreased proliferation rate suggests that cellular immune function is suppressed. In a pig model, Nguyen et al. demonstrated decreased lymphocyte proliferation after both open and laparoscopic cholecystectomy; however, the open group was significantly more suppressed than the minimally invasive group [11]. Horgan et al. carried out an LPA study on mice undergoing sham full laparotomy, $\mathrm{CO}_{2}$ pneumoperitoneum, or anesthesia alone [12]. Proliferation was significantly depressed postoperatively in the open group mice only. Lee et al., also in mice, confirmed the Horgan et al. results but also determined the time course of the depressed lymphocyte proliferation following laparotomy [13]. 
Significantly decreased proliferation rates were noted on postoperative days 2 , 3 , and 4 whereas the results had normalized by day 8 (the next sampling point). Griffith et al., in a nonrandomized human study, showed that there was significant reduction in lymphocyte proliferation the day after open cholecystectomy when compared to the results after laparoscopic removal of the gallbladder [14]. To summarize, the small number of animal and human LPA studies that have been done demonstrate short-lived, relatively small, yet statistically significant differences in proliferation rates between open and closed surgery groups. These results suggest that the lymphocytes from laparoscopic patients may be more easily activated early after surgery than those from open patients.

\section{Lymphocyte Subpopulation Studies}

Studies assessing the number of circulating lymphocytes of different subtypes have, with rare exception, found no significant differences between open and closed groups $[9,15,16]$. A randomized cholecystectomy study that indirectly assessed the ratio of Th-1/Th-2 lymphocytes by measuring levels of interferon- $\gamma$ (Th-1) and interleukin-4 (IL-4) (Th-2) that were elaborated by peripheral blood monocytes in vitro, after stimulation, found a significant difference between the laparoscopic and open groups only 2 hours after the operation; all other sampling points yielded similar result between groups [16]. They suggested that this shift in cytokine release was a manifestation of downregulated cell-mediated immunity and upregulated humoral immunity. A recent colectomy study analyzed CD31 expression on circulating T lymphocytes before and after surgery. Efficient killing of tumor cells or other pathogens depends on, among other things, T-cell migration from the circulation to peripheral tissues. $\mathrm{T}$ cells migrating from the circulation to the peripheral tissues express the CD31 antigen. CD31 expression was found to be significantly decreased from preoperative baseline levels in the open group but not in the laparoscopic group. Furthermore, there was a significant correlation between the decrease in CD31 expression and the incision length in the open group [17]. These results suggest that there are more circulating $\mathrm{T}$ cells able to migrate to the periphery after laparoscopy than following open surgery.

\section{Monocytes and Neutrophils}

Monocytes, which play a central role in cell-mediated immune function, have also been studied perioperatively. Monocytes present antigens to lymphocytes in the context of major histocompatibility (MHC) restriction via class II human leukocyte antigen (HLA) molecules such as HLA-DR. Decreases in the percentage of circulating monocytes that express HLA-DR are associated with a worse short-term outcome after major elective surgery and trauma [18-20]. Kloosterman et al. reported a significant decline in the expression of HLA-DR 1 day after open cholecystectomy whereas laparoscopic removal was not associated with a change from baseline [9]. Ordemann and colleagues reported the 
results of a prospective, randomized clinical trial of patients with colorectal cancer [21]. Their group found that both procedures resulted in significant reductions in postoperative HLA-DR expression compared with preoperative levels. When the two groups' results were compared, a significantly lower level of HLADR expressions was noted in the open group only on the fourth postoperative day.

Studies involving in vitro stimulation of peripheral blood mononuclear cells (PBMCs) followed by measurement of cytokine elaboration have also been carried out. Open cholecystectomy has been shown to be associated with significantly higher tumor necrosis factor (TNF)-alpha and superoxide anion elaboration from PBMCs following stimulation. Therefore, PBMCs are more readily activated after the open procedure than after the laparoscopic equivalent. Neutrophil chemotaxis and superoxide elaboration were similarly studied. Similar results were noted; chemotaxis was increased and superoxide elaboration increased after open cholecystectomy [22]. Some investigators argue that these alterations in monocyte and neutrophil responsiveness might be beneficial because these patients would be better prepared to respond to an early bacterial infection. The authors, however, suggest that postoperative overproduction of these inflammatory mediators may lead to a cycle of overproduction of these mediators, which could cause dysfunction of vital organs. Therefore, the clinical relevance of these findings is not clear.

\section{E. Interleukin 6}

The cytokine interleukin 6 (IL-6) is produced by monocytes, fibroblasts, and vascular endothelial cells in response to IL-1 and TNF. IL-6 stimulates hepatocytes to synthesize fibrinogen and thus contributes to the acute-phase response. IL-6 levels are not a measure of immune function; however, they have been used as a means of assessing overall surgical stress. IL-6 levels have been shown to be elevated following major open surgery. The majority of studies comparing laparoscopic and open cholecystectomy have reported significantly lower IL-6 levels in the laparoscopic groups, which suggests that the minimally invasive approach is less stressful. Conflicting results have been noted after colectomy in humans. One study found significantly lower IL-6 levels in the laparoscopic group during the first 12 hours following laparoscopic-assisted colectomy when compared to open group levels. At 24,48 , and 72 hours after surgery no significant differences were observed [23]. Ordemann et al. noted significantly lower levels 1 day after surgery in the laparoscopic group patients. At least three other investigators, none of whom measured IL-6 levels in the first 12 hours after surgery, found no differences [24]. One study that assessed laparoscopic colectomy done via an abdominal wall lifting device found higher levels after the minimally invasive operation. To summarize, presently the impact of open and closed colectomy on IL-6 levels is not clear. There may be a short-lived difference, persisting for less than a day. 


\section{F. Peritoneal Macrophages}

As mentioned earlier, although systemic cell-mediated immunity seems to be less affected by laparoscopic than open surgery, there is evidence that the carbon dioxide pneumoperitoneum may have a detrimental impact on local peritoneal immunity. Peritoneal macrophages function as the immune system's first line of defense in the abdominal cavity. A fair number of small animal studies that assessed peritoneal macrophage function after carbon dioxide pneumoperitoneum have reported diminished macrophage cytokine release and impaired ability of the macrophages to clear intraperitoneal bacterial loads [25-27]. Other animal studies, however, have reported conflicting results. There are few human data available, and the animal data remain confusing. Until further human studies are conducted, no firm conclusions can be made regarding the choice of operative technique and its impact on intraperitoneal immunity.

\section{G. Etiology of Surgery-Related Immunosuppression}

What is it about abdominal surgery that causes temporary suppression of the immune system? Probably there are a number of contributing factors. There is evidence that the overall length of the abdominal wall incision is an important factor. Others, based on the results of a murine study, believe that it is exposure of the abdominal cavity to air that is the cause of the immunosuppression after open surgery. These latter investigators believe that small amounts of lipopolysaccharide (LPS) in the air cause immunosuppression by stimulating bacteria in the intestine to elaborate LPS, which then translocates across the bowel wall, after which it is absorbed systemically [28].

\section{H. Perioperative Immunomodulation}

Is it possible to avoid surgery-related immunosuppression pharmacologically? A single small randomized study determined the impact of daily perioperative injections of granulocyte-macrophage colony-stimulating factor (GM-CSF) on immune function after open colectomy [29]. It was demonstrated that such treatment is associated with significantly better preserved postoperative DTH responses and monocyte HLA-DR expression levels than following surgery alone. It remains to be proven that this treatment will result in a lower rate of perioperative complications or improved short-term outcome. Although there are no data thus far, in theory, this type of treatment might influence oncologic outcome. Numerous animal studies, utilizing different models, have determined the impact of perioperative immunomodulation on several oncologic variables. These studies have demonstrated that such treatment decreases the rate of tumor growth and establishment as well as the rate of tumor metastasis formation [30-33]. This line of research would seem to warrant further investigation. 


\section{Summary}

To summarize, the results of the DTH studies, the lymphocyte proliferation studies, and select lymphocyte and monocyte function studies suggest that open surgery causes more systemic immunosuppression than laparoscopic procedures. However, there are other studies, concerning a limited number of immune function indicators, that have found no significant differences in immune function between the two surgical methods. There are still other studies that have yielded results which are not easily interpreted. Although the overall interpretation of this literature is open to discussion, it is the authors' belief that there are sufficient data to support the position that minimally invasive surgery is associated with better-preserved immune function than that following traditional open surgical methods. Finally, there are some data to suggest that surgery-related immunosuppression may be avoided via perioperative immunomodulation for open surgery patients.

\section{J. References}

1. Nielsen HJ, Pedersen BK, Moesgaard F. Effect of Ranitidine on postoperative suppression of natural killer cell activity and delayed hypersensitivity. Acta Chir Scand 1989;155:377-382.

2. Christou NV, Superina R, Broadhead M, et al. Postoperative depression of host resistance: determinants and effect of peripheral protein-sparing therapy. Surgery 1982; 92:786-792.

3. Nielsen HJ, Moesgaard F, Kehlet H. Ranitidine for prevention of postoperative suppression of delayed hypersensitivity. Am J Surg 1989;157:291-294.

4. Hjortso NC, Kehlet H. Influence of surgery, age, and serum albumin on delayed hypersensitivity. Acta Chir Scand 1986;152:175-179.

5. Lennard TW, Shenton BK, Borzotta A, et al. The influence of surgical operations on components of the human immune system. Br J Surg 1985;72:771-776.

6. Lacy AM, Garcia-Valdecasas JC, Delgardo S, et al. Laparoscopically-assisted colectomy versus open colectomy for treatment of non-metastatic colon cancer: a randomised trial. Lancet 2002;359:2224-2229.

7. Trokel MJ, Bessler M, Treat MR, et al. Preservation of immune response after laparoscopy. Surg Endosc 1994;8:1385-1388.

8. Allendorf JD, Bessler M, Whelan RL, et al. Better preservation of immune function after laparoscopic-assisted versus open bowel resection in a murine model. Dis Colon Rectum 1996;39:67-72.

9. Kloosterman T, von Blomberg ME, Borgstein $\mathrm{P}$, et al. Unimpaired immune functions after laparoscopic cholecystectomy. Surgery 1994;115:424-428.

10. Whelan RL, Franklin M, Donahue J, et al. Postoperative cell mediated immune response is better preserved after laparoscopic versus open colectomy in humans: a preliminary study. Surg Endosc 1998;12:551. 
11. Nguyen NT, Luketich JD, Schatz S, et al. Effect of open and laparoscopic surgery on cellular immunity in a swine model. Surg Laparosc Endosc Percutan Tech 1999;9: 177-180.

12. Horgan PG, Fitzpatrick M, Couse NF, et al. Laparoscopy is less immunotraumatic than laparotomy. Minim Invasive Ther 1992;1:241-244.

13. Lee SW, Southall JC, Gleason NR, et al. Time course of differences in lymphocyte proliferation rates after laparotomy versus $\mathrm{CO}_{2}$ insufflation. Surg Endosc 2000;14: $145-148$.

14. Griffith JP, Everitt NJ, Lancaster F, et al. Influence of laparoscopic and conventional cholecystectomy upon cell-mediated immunity. Br J Surg 1995;82:677-680.

15. Perttila J, Salo M, Ovaska J, et al. Immune response after laparoscopic and conventional Nissen fundoplication. Eur J Surg 1999;165:21-28.

16. Decker D, Schöndorf M, Bidlingmaier F, et al. Surgical stress induces a shift in the type-1/type-2 T-helper cell balance, suggesting down-regulation of cell-mediated and up-regulation of antibody-mediated immunity commensurate to the trauma. Surgery 1996;119:316-325.

17. Kirman I, Cekic V, Asi Z, et al. The percentage of CD31+ T-cells decreases after open but not laparoscopic surgery in humans. Surg Endosc 2002;16(suppl 1):s192.

18. Appel SH, Wellhausen SR, Montgomery R, et al. Experimental and clinical significance of endotoxin independent HLA-DR expression on monocytes. J Surg Res 1089; 47:44-48.

19. Faist E, Mewes A, Strasser T, et al. Alteration of monocyte function following major injury. Arch Surg 1988;123:287-292.

20. Hershman MJ, Cheadle WG, Wellhausen SR, et al. Monocyte HLA-DR antigen expression characterizes clinical outcome in the trauma patient. Br J Surg 1990;77: 204-207.

21. Ordemann J, Jacobi A, Schwenk W, et al. Cellular and humoral inflammatory response after laparoscopic and conventional resections. Surg Endosc 2001;15:600-608.

22. Redmond HP, Watson WG, Houghton T, et al. Immune function in patients undergoing open vs. laparoscopic cholecystectomy. Arch Surg 1994;129:1240-1246.

23. Harmon GD, Senagore AJ, Kilbride MJ, Warzynski MJ. Interleukin-6 response to laparoscopic and open colectomy. Dis Colon Rectum 1994;37:754-759.

24. Mehigan BJ, Hartley JE, Drew PJ, et al. Changes in T cell subsets, interleukin-6, and C-reactive protein after laparoscopic and open colorectal resection for malignancy. Surg Endosc 2001;15:1289-1293.

25. Neuhaus SJ, Watson DI, Ellis T, et al. Influence of gases on intraperitoneal immunity during laparoscopy in tumor-bearing rats. World J Surg 2000;24:1227-1231.

26. Chekan EG, Nataraj C, Clary EM, et al. Intraperitoneal immunity and pneumoperitoneum. Surg Endosc 1999;13:1135-1138.

27. West MA, Hackam DJ, Baker J, et al. Mechanism of decreased in vitro murine macrophage cytokine release after exposure to carbon dioxide: relevance to laparoscopic surgery. Ann Surg 1997;226:179-190.

28. Watson RWG, Redmond HP, McCarthy J, Burke PE, Hayes DB. Exposure of the peritoneal cavity to air regulates early inflammatory responses to surgery in a murine model. Br J Surg 1995;82:1060-1065. 
29. Mels AK, Statius Muller MG, van Leeuwen PAM, et al. Immune-stimulating effects of low-dose perioperative recombinant granulocyte-macrophage colony stimulating factor in patients operated on for primary colorectal carcinoma. Br J Surg 2001; 88:539-544.

30. Schuurman B, Heuff G, Beelen RHJ, et al. Enhanced killing capacity of human Kupffer cells after activation with granulocyte/macrophage-colony-stimulating factor and interferon-?. Cancer 1994;39:179-184.

31. Tanemura H, Sakata K, Kunieda T, et al. Influences of operative stress on cellmediated immunity and on tumor metastasis and their prevention by nonspecific immunotherapy: experimental studies in rats. J Surg Oncol 1982;21:189-195.

32. Heys SD, Deehan DJ, Eremin O. Interleukin-2 treatment in colorectal cancer: current results and future prospects. Eur J Surg Oncol 1994;20:622-629.

33. Hill ADK, Redmond HP, Naama HA, et al. Granulocyte-macrophage colonystimulating factor inhibits tumor growth during the postoperative period. Surgery 1996;119:178-185. 